\title{
How to make antimatter last
}

\section{An experiment at CERN has shown that antiprotons can be kept for several minutes in an electro- magnetic trap. But the prospect of making reality of the science fiction remains remote.}

SCIENCE fiction writers, ingenious people as they are, include among the standard props of their trade a ready supply of antimatter, the most portable of all highexplosives. During the past few years, there has also been a disturbing trickle of accounts in the popular press suggesting that the world's accelerator laboratories are hard at work on the development of techniques for making antimatter for use in military operations. A glance at one of the first published accounts of success in storing antiprotons (Phys. Rev. Lett. 57, 2504: 1986) should persuade all those concerned that it will be some time before the fiction can become reality.

For other reasons, the new development is interesting and important. Working at CERN, the European high-energy physics laboratory at Geneva, a group from the University of Washington at Seattle (Gabrielse, Kei, Helmerson, Rolston, Tjoekler and Trainor), two people from the University of Mainz (Kalinowsky and Haas) and one from the Fermi National Accelerator Laboratory (Wells) have been able to decelerate antiprotons to the point at which their energy is sufficiently small to allow them to be captured in an electromagnetic trap. The experiment is a technical achievement in its own right, but the prospect that it will now be possible to study antiprotons essentially at rest, and stable for some minutes, raises the prospect of a series of previously impossible experiments.

CERN's advantage in this connection is the facility called the Low Energy Antiproton Ring (LEAR), which is a device for storing bunches of antiprotons produced by the Super Proton Synchrotron (as modified so that protons and antiprotons travel in opposite directions) and reducing their individual energy by four orders of magnitude to $5 \mathrm{MeV}$ or thereabouts. Novel experiments are possible even at this energy (see Batty, C.J., News \& Views, Nature 323, 487; 1986). But even 5 million volts is far greater than the electrostatic potential well of the kinds of traps that might be constructed in the laboratory.

The obvious way to make possible the trapping of antiprotons would be a further elaboration of the LEAR storage ring, but although CERN has ambitions in that direction, the extra equipment has not yet been built. Accordingly, the WashingtonMainz group has fallen back on the simplest of all devices for robbing fast charged particles of energy - that of making them traverse a dense target of matter, a solid beryllium window. In practice, the experiment uses virtually instantaneous bunches of antiprotons, each of them containing between 10 million and 1,000 million particles.

Inevitably, the process is inefficient. With the thickness of the window chosen to be the range of antiprotons, at the energy discharged from LEAR, in beryllium, only about 1 in 10,000 of the original bunch emerges from the target with an energy less than $3 \mathrm{keV}$, which means that typical bunches leave only some 10,000 antiprotons for catching in a trap.

That consists of two components, of which the simplest is a powerful axial magnetic field to prevent slow antiprotons moving too quickly away from the axis of the experiment. The other is a conceptually simple but technically sophisticated pair of electrodes, each with a hole in the centre (a third of a centimetre across) to allow the passage of the antiproton beam, and roughly a dozen centimetres apart. Before the arrival of an antiproton bunch, the first electrode is at earth potential and the second, or more distant, has a potential of $-3 \mathrm{kV}$ so as to repel antiprotons less energetic than $3 \mathrm{keV}$. But while the bunch is travelling the length of the trap, the voltage of the first electrode is switched, in a matter of nanoseconds, to match the potential of the first.

For the time being, the objective is to see how efficient such a simple trapping technique may be. As these things go, the mechanism seems to work as well as anybody expected in advance. The population of antiprotons can be measured at any time by abolishing the repulsive potential on the more distant of the two electrodes, whereupon the antiprotons are guided by the axial magnetic field to a target where they annihilate, yielding pions which can be counted. Apparently, the counting equipment is not yet fast enough to measure the population of a newly filled trap, but typical records show that, after a millisecond, the trap may contain 300 or so antiprotons. One of the published records shows that after storage for 100 seconds, it was still possible to record 31 antiprotons, spread over a mere 5 microseconds. On balance, it seems as if it is possible to catch roughly 3 in a million of all the particles discharged as a bunch from LEAR.

At this rate, sheer numbers are less important than the other properties of the trap, which will determine what use can be made of a supply of essentially static antiprotons. The authors of the experiment brim over with suggestions for what may happen next. One scheme is to make single atoms of anti-hydrogen, perhaps by building two traps alongside each other, one for antiprotons and one for positrons. Firing a beam of positronium atoms (pairs of positive and negative electrons) at antiprotons in a trap might be a simpler way of accomplishing the same goal. The prospect of precision measurements of quantities such as the antiproton mass, or even of the gravitational acceleration of antiprotons, will intrigue many observers of this new development.

Technically, the improvement of the present means of trapping antiprotons is an obvious line of evolution, but the authors write of the still more promising line of development in which antiprotons trapped as they describe would be transferred, bunch by bunch, to a trap whose geometry is not constrained by the need to capture as many particles as possible from LEAR. Such a device would therefore be better suited for keeping even these unstable particles for long periods of time, a day or even longer. That is what will have to be done if some of the more interesting measurements now suggested are to be carried out.

All this is of course a long way from what the science fiction writers require of antimatter. While the best that people can do is to store a few hundred antiprotons for an hour or so, the prospect of antimatters weapons will remain very distant. The essential difficulty is a score or so of orders of magnitude. True, the annihilation of an antiproton by, say, a proton of the kind that turns up in ordinary matter is more energetic, by a factor of say a hundred, than the fission of a uranium nucleus. But Avogadro's number $\left(6.10^{23}\right)$ works emphatically in the other direction. So long as numbers of antiprotons that people can collect in antiproton traps are small enough that it is feasible to think of counting them, the popular newspapers' accounts of what high-energy physics is really for will remain in the realm of fantasy. It is nevertheless outrageous that there have been in the past few years, such a torrent of tales along these lines. They will always be fairy stories. So, too, perhaps sadly, will be the relevant parts of science fiction.

John Maddox 